\title{
Unilateral Tonsillar Swelling as a Manifestation of Diffuse Large B Cell Lymphoma (DLBCL): Case Report
}

Wulyo RAJABATOa, Vitya CHANDIKAa, Agnes Stephanie HARAHAPb

aDivision of Hematology-Medical Oncology, Department of Internal Medicine, Dr. Cipto Mangunkusumo General Hospital, Faculty of Medicine Universitas Indonesia

bDepartment of Anatomical Pathology,

Dr. Cipto Mangunkusumo General Hospital/ Faculty of Medicine Universitas Indonesia

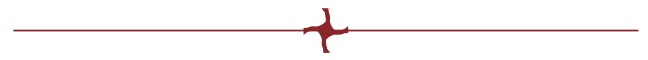

\begin{abstract}
Approximately $30-40 \%$ of all diffuse large B-cell lymphoma (DLBCL) cases, which have initially been present in extranodal sites with the most common involvement, were found at the level of the gastrointestinal tract $(34 \%)$, head and neck (13.6\%), and skin/soft tissue (10.6\%). More than half of head and neck lymphomas occur in the Waldeyer ring, with $40 \%$ to $50 \%$ of these arising from the tonsil. The majority of tonsil lymphomas are B-cell origin, and DLBCL is the most common histological type. Here we presented a case of tonsillar DLBCL with long-term complete remission after R-CHOP treatment.

Keywords: unilateral tonsillar swelling, DLBCL CD20 positive, chemotherapy R-CHOP, long-term complete remission.
\end{abstract}

\section{INTRODUCTION}

The majority of DLBCLs originate in lymph nodes; however, $30-40 \%$ of all cases initially present in extranodal sites $(1,2)$. The head and neck area is the second most common site of extranodal lymphoma, with tonsils being the most common site of involvement; other sites include the nasopharynx and tongue base. Diffuse large $\mathrm{B}$-cell lymphoma is the most frequent histologic subtype (2). These lymphomas occur predominantly in elderly males and present as tonsillar swelling, cervical adenopathy, dysphagia, odynophagia, or a sore throat (3). Here, we reported a case of unilateral tonsillar swelling as a manifestation of DLBCL.

\section{CASE DESCRIPTION}

59-year-old male with a good Eastern Cooperative Oncology Group (ECOG) performance status complained of a sore throat for one month. He also felt dysphagia, odynophagia, and unusual snoring. There was no history of weight loss, night sweats, or prolonged fever. There was no comorbidity. Physical examination revealed unilateral swelling of the right tonsil and lymphadenopathy of the right mandibula. Cervical computed tomography (CT) scan showed a solid tumor mass of the right palatine tonsil with

\section{Address for correspondence:}

Vitya Chandika

Division of Hematology-Medical Oncology, Department of Internal Medicine Dr. Cipto, Mangunkusumo General Hospital, Faculty of Medicine Universitas Indonesia

Phone +62819705797

Email vityachandika@gmail.com 
FIGURE 1. CT scan of the nasopharynx
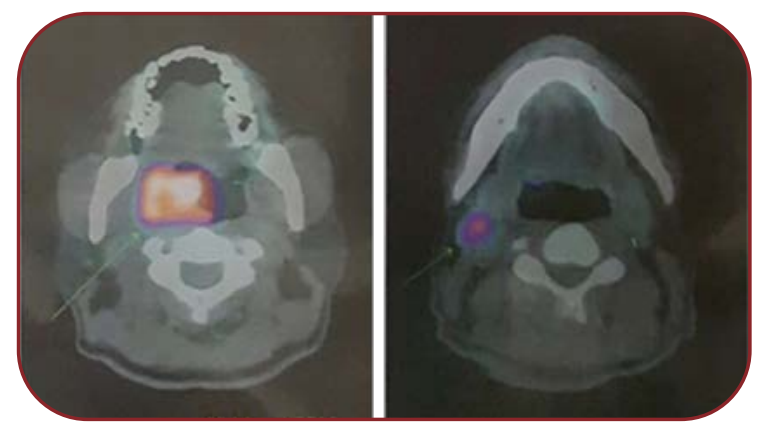

$3.65 \times 3.39 \times 3.88 \mathrm{~cm}$ in diameter and multiple lymph nodes less than $1 \mathrm{~cm}$ at the submandibular level (Figure 1).

His positron emission tomography (PET)/CT (PET/CT) scan presented a hypermetabolic mass of

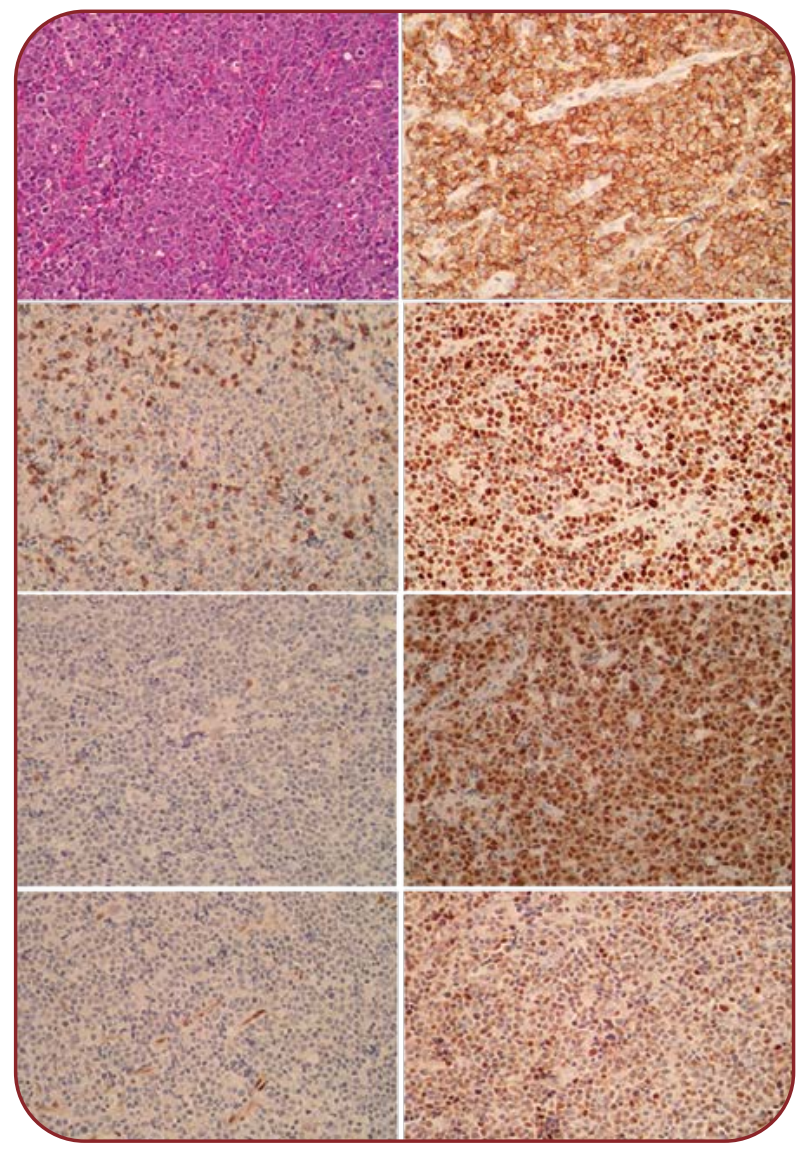

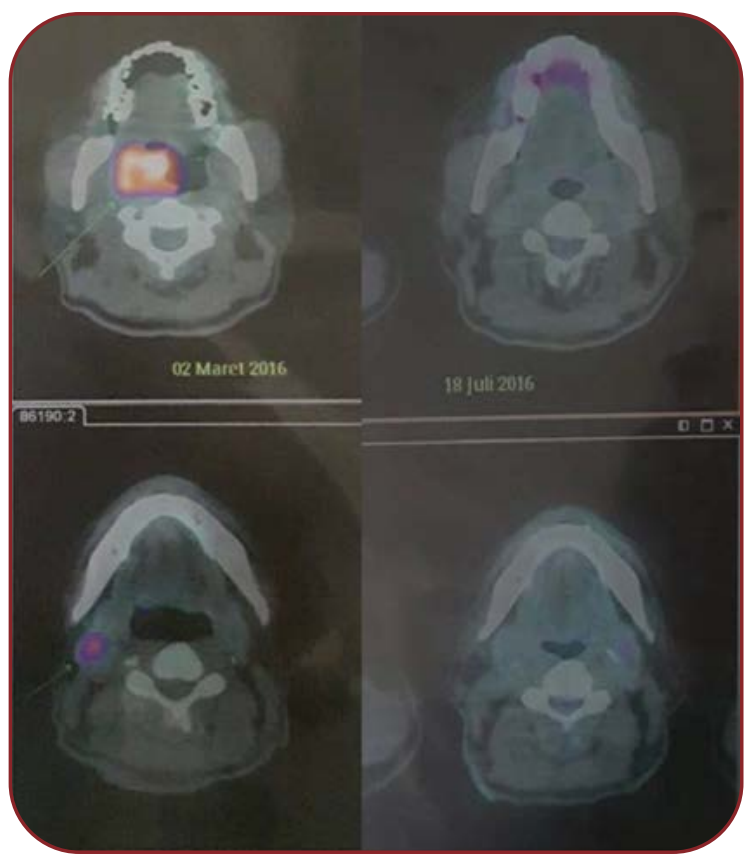

FIGURE 4. PET CT scan. A) before therapy; B) after RCHOP

$4.8 \times 1.9 \mathrm{~cm}$ in the right palatine tonsil and hypermetabolic right submandibular lymph node (Figure 2).

The otolaryngologist performed a biopsy of the right tonsil. Histopathology showed a diffuse proliferation of large-sized tumor cells with pleomorphic nuclei, coarse chromatin and scanty eosinophilic cytoplasm. Immunohistochemistry staining was positive for CD 45, CD 20, BCL6, BCL2 and MUM1, while CD 10 and cyclin D1 were negative. The ki67 proliferation index was high (80-90\%) and therefore confirmed the CD 20 positive non-germinal center B-cell (non-GCB) subtype of DLBC (Figure 3).

We established the diagnosis of $C D 20$ positive DLBCL of the right palatine tonsil, stage $2 \mathrm{AE}$, ageadjusted IPI (aa-IPI) = 0, and administered six cycles of the R-CHOP regimen. Each cycle was three weeks apart. The response rate was complete remission. The patient tolerated the chemotherapy well, although he developed febrile neutropenia once during the first cycle. We performed a PET scan evaluation annually, and the patient was still in complete remission in the fifth year (Figure 4). $\square$

\section{DISCUSSION}

on-Hodgkin lymphoma is primarily a disorder of the lymph nodes (3). However, up to $40 \%$ of all non-Hodgkin lymphomas may arise 
from extranodal sites (1). Castillo et al reported that DLBCL primarily involved the lymph nodes $(68.4 \%)$ and extranodal sites (31.6\%), with the most common extranodal sites of involvement being the gastrointestinal tract (34\%), head and neck (13.6\%), and skin/soft tissue (10.6\%) (4). Furthermore, more than half of head and neck lymphomas occur in the Waldeyer ring, with $40 \%$ to $50 \%$ of them arising from the tonsil besides nasopharynx and tongue base $(3,4)$. Tonsil lymphomas occur predominantly in elderly males and present as tonsillar swelling, cervical adenopathy, dysphagia, odynophagia, or a sore throat. The majority of tonsil lymphomas originate from B-cells, and DLBCL is the most common histological type (3). Diagnosis of lympho$\mathrm{ma}$ is based on the histopathology result. Immunohistochemical staining in biopsy material allows to differentiate forms of lymphoma and from anaplastic neoplasm. Disease staging is completed with imaging evaluation. Positron emission tomography completes the CT evaluation as it provides metabolic activity information (5).

Our patient was younger than reported in the literature and presented with unilateral right tonsillar swelling with submandibular lymphadenopathy, complaining about a sore throat, dysphagia, odynophagia, unusual snoring, and even histopathology with immunohistochemistry revealed CD20 positive DLBCL.

According to the age-adjusted international prognostic index (aa-IPI) in patients $\leq 60$ years based on risk factors, our patient had a IIAE stage of DLBCL, normal serum LDH and $100 \%$ performance status. Therefore, he was considered to belong to the low risk category, with an estimated three-year overall survival (OS) of 98\% (6). Com- pared to nodal DLBCL, patients with extranodal involvement presented with earlier stages just like our patient. Castillo et al found that primary extranodal sites of disease were associated with good prognosis in patients with DLBCL in the rituximab era and reported that, based on multivariate analysis, the head and neck region was the site of involvement associated with a better survival (HR $0.79,95 \% \mathrm{Cl} 0.70-0.89 ; \mathrm{P}<0.001)$ compared to gastrointestinal, pulmonary, and liver/pancreas localization (4). Our tonsillar DLBCL patient aged under 60, presenting with early-stage disease and age-adjusted IPI low risk, has already achieved five-year OS without disease progression. CHOP has been used as the standard therapy for DLBCL. Addition of rituximab, an antibody against CD20, to the regimen was proved to ensure prolonged event-free survival (EFS) and OS in the R-CHOP treated group (7).

Currently, six R-CHOP cycles are considered as standard therapy for young low risk patients $($ aa-IPI=0) $(1,7)$. Given that our patient was a low risk person, we decided to administer six cycles of $\mathrm{R}-\mathrm{CHOP}$.

\section{CONCLUSION}

Primary lymphoma of the tonsil is a rare entity. Diffuse large B-cell lymphoma is the most commonly found histological subtype. Chemotherapy $\mathrm{R}-\mathrm{CHOP}$ is the treatment option for tonsillar lymphoma. Since our aa-IPI patient was a low risk person, treatment with six cycles of R-CHOP showed a good prognosis.

Conflicts of interest: none declared. Financial support: none declared.

\section{$\mathbf{R}_{\text {EFERENCES }}$}

1. Vitolo U, Seymour JF, Martelli M, et al. Extranodal diffuse large B-cell lymphoma (DLBCL) and primary mediastinal B-cell lymphoma: ESMO clinical practice guidelines for diagnosis, treatment, and follow-up.

Ann Oncol 2016;27(Suppl 5):v91-v102.

2. Vannata B, Zucca E. Primary extranodal B-cell lymphoma: current concepts and treatment strategies.

Chin Clin Oncol 2015;4:1-17.

3. Laskar S, Bahl G, Muckaden MA.
Primary diffuse large B-cell lymphoma of the tonsil: is a higher dose radiotherapy required?

Cancer 2007;110:816-823.

4. Castillo JJ, Winer ES, Olszewski AJ. Sites of extranodal involvement are prognostic in patients with diffuse large B-cell lymphoma in the rituximab era: An analysis of the surveillance, epidemiology, and end results database. Am J Hematol 2014;89:310-314.

5. Zapater E, Bagán JV, Carbonell F,
Basterra J. Malignant lymphoma of the head and neck. Oral Dis 2010;16:119-128.

6. Tilly H, Da Silva G, Vitolo U, et al. Diffuse large B-cell lymphoma (DLBCL): ESMO clinical practice guidelines for diagnosis, treatment, and follow up.

Ann Oncol 2015;26 (Suppl 5):v116-v125.

7. Kwak JY. Treatment of diffuse large B cell lymphoma.

Korean J Intern Med 2012;27:369-377. 